\title{
Tratamiento de la osteoporosis postmenopáusica
}

\section{Treatment of postmenopausal osteoporosis}

\section{E. Loza}

\section{RESUMEN}

El tratamiento de la osteoporosis en mujeres postmenopáusicas consiste en medidas farmacológicas y no farmacológicas. La terapia hormonal sustitutiva hasta épocas recientes era considerada una terapia de primera línea para la prevención de la osteoporosis postmenopáusica. Sin embargo, los datos derivados del estudio Women's Health Initiative (WHI) revelaron que el tratamiento combinado estrógenos-progestágenos no reduce el riesgo de enfermedad coronaria sino que además aumenta el riesgo de cáncer de mama, ictus y eventos tromboembólicos venosos. Por este motivo actualmente el tratamiento de elección de la osteoporosis postmenopáusica lo constituyen otros agentes antiresortivos.

Palabras clave. Osteoporosis postmenopáusica. Tratamiento. Bifosfonatos. Raloxifeno. Calcitonina.

\begin{abstract}
Prevention and treatment of osteoporosis consists of non-drug and drug therapy. This review of the topic will provide an overview of the approach to therapy of osteoporosis in postmenopausal women. In the past, estrogen replacement was considered a primary therapy for the prevention of postmenopausal osteoporosis. However, data from the Women's Health Initiative (WHI) revealed that estrogen-progestine therapy does not reduce the risk of coronary heart disease, and increases the risk of breast cancer, stroke and venous thromboembolic events. As a result of these findings, other antiresorptive agents are now the drugs of choice for the prevention and treatment of osteoporosis in postmenopausal women.
\end{abstract}

Key words. Postmenopausal osteoporosis. Treatment. Bisphosphonates. Raloxifene. Calcitonin.

An. Sist. Sanit. Navar. 2003; 26 (Supl. 3): 91-98.

Sección de Reumatología. Hospital de Navarra. Pamplona.

\author{
Correspondencia: \\ Dr. Eduardo Loza Cortina \\ Sección de Reumatología \\ Hospital de Navarra \\ C/ Irunlarrea, 3 \\ 31008 Pamplona \\ Tfno. 848422649 \\ Fax 848422340
}




\section{INTRODUCCIÓN}

El tratamiento de la osteoporosis en mujeres postmenopáusicas, consiste en medidas farmacológicas y no farmacológi$\operatorname{cas}^{1}$ (Tabla 1).

La terapia hormonal sustitutiva hasta épocas recientes era considerada una terapia de primera línea para la prevención de la osteoporosis postmenopáusica. Sin embargo, los datos derivados del estudio Women's Health Initiative (WHI) revelaron que el tratamiento combinado estrógenosprogestágenos no reduce el riesgo de enfermedad coronaria sino que además aumenta el riesgo de cáncer de mama, ictus y eventos tromboembólicos veno$\operatorname{sos}^{2}$. Por este motivo actualmente el tratamiento de elección de la osteoporosis postmenopáusica lo constituyen otros agentes antiresortivos.

\section{TERAPIA NO FARMACOLÓGICA}

La terapia no farmacológica incluye la dieta, el ejercicio y el abandono del consumo de tabaco.

\section{Dieta}

Una dieta óptima incluye una adecuada ingesta de calorías (para evitar mal nutrición), calcio y vitamina D.

\section{Calcio}

La mujer postmenopáusica debería ingerir suplementos adecuados de calcio elemento (generalmente 1.000-1.500 $\mathrm{mg} /$ día) con las comidas y en dosis divididas, de tal manera que la ingesta total de calcio, incluida la aportada por los alimentos, se aproxime a los $1500 \mathrm{mg} /$ día $^{3}$. Un adecuado aporte de calcio reduce la pérdida de masa ósea en adultos ${ }^{4}$. La mayor fuente de calcio se encuentra en los productos lácteos; un litro de leche contiene aproximadamente un gramo de calcio elemento (un yogur aproximadamente 125 $\mathrm{mg}$ ). La cantidad de calcio aportada en la dieta fuera de los productos lácteos no supera los $300 \mathrm{mg}$ por día.

En los pacientes con una dieta inadecuada en calcio se recomienda aportar suplementos más que modificaciones en la dieta (probablemente difíciles de cumplir).

A la hora de aportar suplementos de calcio hay que tener en cuenta varias consideraciones, como son el tipo de sal de calcio, el tiempo de administración y la dosis.

Las sales más utilizadas son el citrato de calcio y el carbonato de calcio (la forma más barata). La absorción del citrato de calcio no depende del PH gástrico por lo que puede ser administrado independientemente de las comidas ${ }^{5}$; en cambio la absorción del carbonato de calcio depende del PH gástrico motivo por el cual se aconseja administrarlo después de las comidas. Muchos productos de carbonato cálcico presentan una solubilidad escasa

Tabla 1. Prevención y tratamiento de la osteoporosis postmenopáusica.

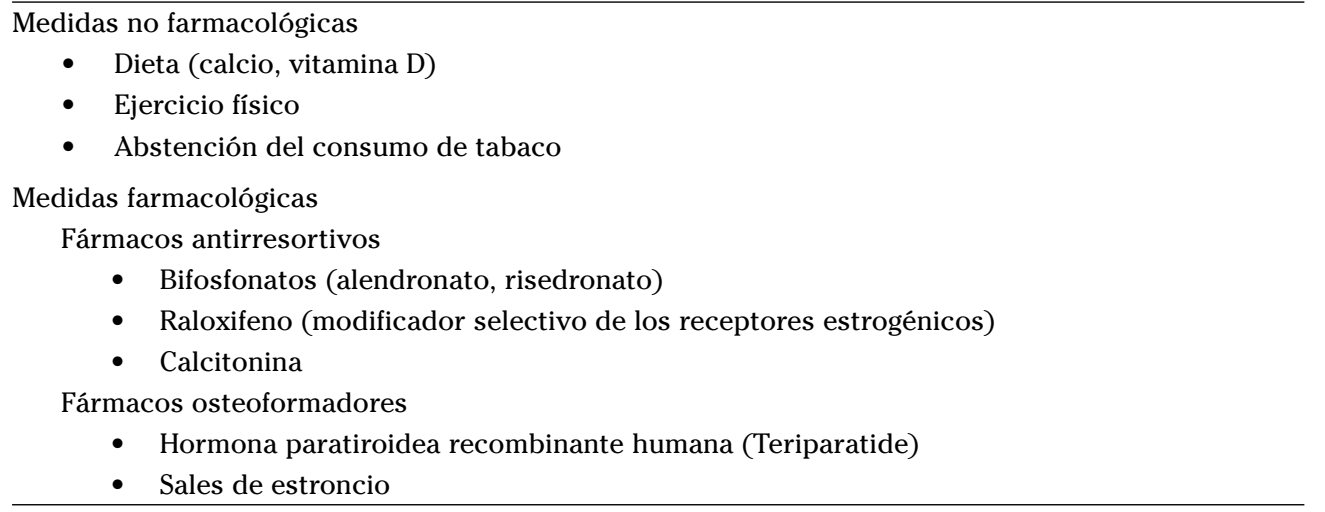


por lo que se recomiendan los preparados masticables.

Cuando se utilizan dosis de calcio elemento mayores de $500 \mathrm{mg}$ se recomienda administrarlas en dosis divididas (cada 12 horas), dosis únicas mayores se asocian con una saturación de la absorción y la consiguiente disminución de la misma ${ }^{6}$.

Los únicos efectos secundarios conocidos relacionados con una alta ingesta de calcio son el estreñimiento y la dispepsia.

El temor al aumento del riesgo de nefrolitiasis en pacientes por otro lado sanos parece infundado ${ }^{7}$.

Los pacientes con malabsorción o síndrome de intestino corto pueden requerir dosis mayores debido a la menor absorción del calcio; esto puede ocurrir incluso en pacientes gastrectomizados. La dosis óptima de calcio en estas situaciones debe ser determinada empíricamente y debe ser ajustada en orden a normalizar la concentración sérica de calcio, fósforo, fosfatasa alcalina, calcidiol, hormona paratiroidea y la excreción de calcio en orina de 24 horas $^{8}$.

Por último hay que tener en cuenta que los diuréticos pueden modificar el balance del calcio. Los diuréticos de asa aumentan la excreción de calcio en tanto que las tiazidas poseen un efecto hipocalciúrico que puede ser protector frente a la aparición de la pérdida de masa ósea.

\section{Vitamina $D$}

Una de las funciones de la vitamina $\mathrm{D}$ es estimular la absorción intestinal de calcio. Los déficit subclínicos de vitamina $\mathrm{D}$ son frecuentes y pueden contribuir al desarrollo de osteoporosis. Los depósitos de vitamina $\mathrm{D}$ dependen de dos factores, la producción endógena derivada de la síntesis cutánea inducida por la exposición a la luz solar y la ingesta de vitamina $\mathrm{D}$ en la dieta. En personas mayores es frecuente encontrar un déficit de ambos factores ${ }^{9,10}$, de manera que es fundamental asegurar un aporte adecuado de esta vitamina.

La ingesta diaria de vitamina $\mathrm{D}$ debería ser al menos de $800 \mathrm{UI}^{11}$. La leche constituye la mayor fuente de vitamina D aportada por la dieta, un litro de leche contiene aproximadamente 400 UI de vitamina D.

En los pacientes con osteoporosis se recomiendan suplementos de vitamina $D$, generalmente a razón de 800 UI diarias. En el mercado existen preparados combinados de calcio y vitamina $\mathrm{D}$, lo cual facilita su administración.

\section{Ejercicio}

La práctica regular de ejercicio en mujeres postmenopáusicas ha demostrado que incrementa la masa ósea y disminuye el riesgo de fracturas de cadera ${ }^{12}$. Caminar 30 minutos diarios parece suficiente.

\section{Abandono del consumo de tabaco}

Se recomienda el abandono del uso del tabaco, ya que acelera la pérdida de masa ósea. En un estudio realizado en mujeres gemelas discordantes en el uso del taba$\mathrm{co}^{13}$ el consumo de 20 cigarrillos diarios se asoció con una reducción del 5 al $10 \%$ en la densidad ósea. El efecto deletéreo del tabaco puede estar mediado porque acelera el metabolismo de los estrógenos, disminuyendo las concentraciones séricas ${ }^{14}$.

\section{TRATAMIENTO FARMACOLÓGICO}

Las medidas comentadas anteriormente deberían de ser aplicadas de manera universal en mujeres postmenopáusicas para reducir la pérdida de masa ósea. Las mujeres postmenopáusicas con osteoporosis o con alto riesgo de desarrollarla deben de ser tratadas con medidas farmacológicas que incluyen el uso de bifosfonatos, moduladores selectivos de los receptores de los estrógenos o calcitonina. Como decíamos en la introducción la terapia hormonal sustitutiva a largo plazo es motivo de controversia.

Las mujeres candidatas a ser tratadas son aquellas ya diagnosticadas de osteoporosis o con osteopenia (T score -1 a $-2,5$ DS), ya que estas mujeres poseen un riesgo aumentado de fractura.

\section{Bifosfonatos}

Los bifosfononatos son análogos estables del pirofosfato. Su mecanismo exacto 
de acción es incierto; su efecto neto es sobre los osteoclastos o sus precursores, produciendo un incremento de la muerte celular y un descenso en la resorción ósea. Alendronato y risedronato son los bifosfonatos utilizados en la prevención y/o tratamiento de la osteoporosis postmenopáusica.

\section{Alendronato}

Diferentes estudios han demostrado la eficacia a largo plazo de alendronato en mujeres con osteoporosis ${ }^{15-21}$. Las conclusiones de los diferentes trabajos publicados son que alendronato aumenta la densidad ósea tanto a nivel de hueso cortical (antebrazo y cuello femoral) como trabecular (columna vertebral). La disminución del riesgo de fractura se sitúa en torno a un $48 \%$ al nivel de columna vertebral y un $51 \%$ a nivel de cadera. La eficacia y tolerabilidad de alendronato se mantiene a los 7 años de tratamiento ${ }^{22}$.

Alendronato se utiliza a dosis de $10 \mathrm{mg}$ diarios por vía oral. Administrado en dosis única semanal de $70 \mathrm{mg}$ es igualmente efectivo al tiempo que pueden disminuir sus efectos a nivel gastrointestinal ${ }^{23}$.

En los ensayos clínicos la incidencia de problemas del tracto gastrointestinal superior en mujeres en tratamiento con alendronato diario ${ }^{19}$ o una vez por semana ${ }^{24}$ no fue diferente del grupo placebo. Sin embargo la aparición de esofagitis o úlceras esofágicas puede ocurrir, así como en raras ocasiones la aparición de estenosis esofágica $^{25,26}$.

Para reducir el riesgo de esofagitis y aumentar la absorción del fármaco debe ser ingerido con agua abundante media hora antes del desayuno y permanecer el paciente erguido durante ese tiempo. Problemas del tracto gastrointestinal superior como la acalasia o la estenosis esofágica suponen una contraindicación absoluta para el tratamiento con alendronato. El reflujo gastroesofágico supone una contraindicación relativa.

\section{Risedronato}

Risedronato a dosis de $5 \mathrm{mg} /$ día por vía oral ha demostrado su poder antifractura tanto a nivel de columna vertebral ${ }^{27,28}$ como de cuello femoral ${ }^{29}$, situándose la disminución del riesgo de fractura en torno a un $49-61 \%$ a nivel de cuerpo vertebral y un $47 \%$ a nivel de cuello femoral.

Risedronato ya obtiene un descenso significativo del riesgo de fractura al primer año del tratamiento ${ }^{30}$. Esta rapidez de acción es importante ya que se sabe que hasta un $20 \%$ de las mujeres que desarrollan una fractura vertebral presentarán una nueva fractura vertebral en el plazo de un $\mathrm{año}^{31}$.

Risedronato también es efectivo en prevenir la pérdida de masa ósea en pacientes tratados con glucocorticoides ${ }^{32}$.

El riesgo de efectos a nivel del tracto gastrointestinal superior parece bajo. En un análisis de 9 ensayos clínicos donde se incluyeron 10.068 pacientes que recibieron de forma randomizada risedronato 5 $\mathrm{mg} /$ día o placebo hasta 3 años de tratamiento ${ }^{33}$, la diferencia de efectos adversos del trato gastrointestinal superior no fue diferente entre ambos grupos. Los pacientes con enfermedad gastrointestinal activa no fueron excluidos del estudio, de manera que el riesgo de efectos adversos a nivel esofágico parece ser bajo incluso en pacientes con antecedentes de enfermedad esofágica.

La dosis recomendada de risedronato es de $5 \mathrm{mg}$ diarios. Para mejorar la absorción del fármaco se recomienda administrar risedronato 30 minutos antes del desayuno o al menos 2 horas alejado de las comidas. También es recomendable que el paciente permanezca erguido durante los 30 minutos posteriores a la toma del fármaco.

La dosificación semanal de comprimidos de $35 \mathrm{mg}$, ya disponible en el mercado, ha demostrado poseer un perfil de seguridad y eficacia similares a la toma diaria ${ }^{34}$, siendo las recomendaciones para su administración las mismas.

\section{Raloxifeno}

El clorhidrato de raloxifeno (un modulador selectivo de los receptores estrogénicos) es un benzotiofeno no esteroide que se une a los receptores estrogénicos e inhibe 
la resorción ósea aumentando la densidad mineral ósea sin estimular el endometrio en mujeres postmenopáusicas; es por tanto un agonista parcial de los receptores estrogénicos en tejido óseo y cardiovascular ${ }^{35}$.

Los estudios con raloxifeno han demostrado una reducción del riesgo de fracturas vertebrales en torno al $49 \%$, sin demostrar eficacia antifractura al nivel de cadera $^{36}$. La dosis óptima de raloxifeno es de $60 \mathrm{mg} /$ día, pudiéndose administrar en cualquier momento del día. Los estudios a los 4 años de tratamiento (60 mg/día) han obtenido resultados similares ${ }^{37}$. Entre los efectos adversos cabe destacar la aparición de fenómenos tromboembólicos venosos, edemas periféricos y calambres en las piernas.

\section{Calcitonina}

La calcitonina es un péptido de 32 aminoácidos sintetizado por las células $\mathrm{C}$ del tiroides; al unirse a los receptores de los osteoclastos disminuye su actividad con la consiguiente reducción de la resorción ósea ${ }^{3}$.

Chesnut y col ${ }^{38}$ realizaron el ensayo clínico más amplio con calcitonina para el tratamiento de la osteoporosis. Este estudio demostró el poder antifractura de la calcitonina a nivel vertebral (no de cadera) utilizando calcitonina intranasal de salmón a dosis de $200 \mathrm{UI} /$ día (alternando diariamente la fosa nasal), siendo ésta la dosis y vía de administración recomendada actualmente. La administración intranasal puede producir malestar nasal, náuseas y enrojecimiento facial.

Un efecto adicional de la calcitonina es su poder analgésico; el mecanismo es desconocido pero podría estar mediado a través de un aumento en los niveles de endorfinas $^{39}$. Por este motivo la calcitonina puede ser inicialmente el agente antirresortivo de elección en pacientes con dolor debido a una fractura osteoporótica aguda.

Algunos pacientes se hacen resistentes a la acción de la calcitonina tras su uso prolongado, probablemente debido a al aparición de anticuerpos neutralizantes ${ }^{3}$.
La administración de los suplementos de calcio 4 horas después de la calcitonina con el objeto de evitar una hipotética hipocalcemia (práctica clínica que todavía se observa ocasionalmente) no descansa sobre ninguna evidencia científica.

\section{FUTUROS TRATAMIENTOS}

\section{Hormona paratiroidea}

La hormona paratiroidea (PTH) recombinante humana (teriparatide) ha demostrado su eficacia en el tratamiento de la osteoporosis $^{40}$. La PTH actúa aumentando la formación ósea más que la resorción, por tanto posee un efecto osteoformador. En un ensayo randomizado ${ }^{40}, 1.637$ mujeres postmenopáusicas con fracturas vertebrales previas fueron tratadas con PTH (20 ó $40 \mu \mathrm{g} /$ día por vía subcutánea durante una media de 21 meses) o placebo. En los pacientes tratados con PTH se observó un aumento en la densidad ósea a nivel vertebral y una reducción en el número de nuevas fracturas vertebrales. Las fracturas no vertebrales descendieron. Los principales efectos secundarios fueron náusea, cefalea e hipercalcemia.

Un meta-análisis de los diversos ensayos clínicos publicados ha mostrado que la PTH aumenta la densidad ósea a nivel vertebral y disminuye el riesgo de fractura a nivel vertebral y también posiblemente a nivel no vertebral ${ }^{41}$.

En un estudio comparativo con alendronato se observó que la PTH aumenta un 8,3 \% más la densidad ósea a nivel vertebral y produce un mayor descenso del riesgo de fracturas no vertebrales ${ }^{42}$.

La PTH ha sido aprobada por la Food and Drugs Administration (FDA) para su uso en mujeres y hombres con alto riesgo de fractura, incluyendo aquellos con fractura osteoporótica previa, la presencia de múltiples factores de riesgo o fracaso de otros tratamientos. El tratamiento debe ser reservado a pacientes con alto riesgo debido a la necesidad de una inyección diaria, alto coste y la posibilidad del riesgo de osteosarcoma (observado en ratas que recibieron altas dosis). En nuestro país está a punto de comercializarse. 


\section{Sales de estroncio}

El ranelato de estroncio se define como la unión de un ácido orgánico, el ácido ranélico con 2 átomos de estroncio estable. Se cataloga como un fármaco con acción osteoformadora así como con cierta actividad antiresortiva.

En un ensayo clínico 353 mujeres postmenopáusicas y al menos una fractura vertebral previa fueron randomizadas para recibir ranelato de estroncio $(0,5 ; 1$ ó 2 g/día durante 2 años) o placebo; la densidad mineral ósea aumentó de una manera dosis-dependiente ${ }^{43}$. Las mujeres que recibieron $2 \mathrm{~g} /$ día presentaron un descenso de las fracturas vertebrales a los 2 años. Actualmente se encuentra en investigación con ensayos en fase III en curso.

\section{MONITORIZACIÓN DEL TRATAMIENTO}

Es conocido que hasta la sexta parte de las pacientes que reciben terapia antirresortiva no responden al tratamiento ${ }^{44}$. Es importante conocer el grado de respuesta al tratamiento y detectar las pacientes no respondedoras, en cuyo caso habría que plantearse modificaciones terapéuticas. Para ello se han propuesto varias estrategias.

Unos autores recomiendan repetir la densitometría ósea a los 2 años de iniciar el tratamiento; en caso de no respuesta (descenso de la densidad mineral ósea) habría que valorar un cambio terapéutico. Sin embargo hay quien opina que esperar 2 años es demasiado tiempo para detectar a las pacientes no respondedoras.

Otros autores recomiendan monitorizar el tratamiento combinando la realización de densitometría ósea y la determinación de un marcador de resorción ósea. En este caso se realizaría de forma basal una densitometría ósea y un marcador de resorción. Después de 3 meses de tratamiento se obtiene una nueva determinación del marcador de resorción ósea, si los niveles del marcador de resorción disminuyen adecuadamente (un 30 a 50\%), probablemente el tratamiento está siendo eficaz en cuyo caso se continuará durante 2 años, momento en el cual se repetirá la densitometría ósea.

\section{CONSIDERACIONES FINALES}

En la práctica clínica la elección del tratamiento dependerá de diferentes factores como la edad, morbilidad concomitante, seguridad y tolerabilidad del fármaco, preferencias del paciente y obviamente la eficacia antifractura demostrada por la droga en cuestión ${ }^{45,46}$. Los criterios para la elección del tratamiento no están claros. El hecho conocido de que los beneficios del tratamiento no se mantienen tras la suspensión del mismo y que reducciones significativas del riesgo de fractura pueden alcanzarse al año del tratamiento ha generado una tendencia a actuar de manera más rápida en individuos de alto riesgo. Por otro lado la dosificación y duración del tratamiento no está completamente establecida, siendo aún materia de futuras investigaciones.

\section{BIBLIOGRAFÍA}

1. Osteoporosis prevention, diagnosis, and therapy. NIH Conensus Development Panel on Osteoporosis Prevention, Diagnosis, and Therapy. JAMA 2001; 285: 785-795.

2 . Risks and benefits of estrogen plus progestin in healthy postmenopausal women: principal results from the Women's Health Initiative randomized controlled trial. JAMA 2002; 288: 321-333.

3. EASTELL R. Treatment of postmenopausal osteoporosis. N Eng J Med 1998; 338: 736746.

4. Riggs BL, O’Fallon WM, Muhs J, O’CCONOR MK, Kumar R, Melton LJ. Long-Term effects of calcium supplementation on serum parathyroid hormone level, bone turnover, and bone loss in elderly women. J Bone Miner Res 1998; 13: 168-174.

5. Heller HJ, Greer LG, Haynes SD, PoindeXter JR, PAK CY. Pharmacokinetic and pharmacodynamic comparison of two calcium supplements in postmenopausal women. J Clin Pharmacol 2000; 40: 12371243.

6. HARvey JA, Zobitz MM, PAK CY. Dose dependency of calcium absorption. A comparison of calcium carbonate and calcium citrate. J Bone Miner Res 1988; 3: 253-258. 
7. Curhan GC, Willet WC, Speizer FE, Spiegelman D, StANPFER MJ. Comparison of dietary calcium with supplemental calcium and other nutrients as factors affecting the risk for kidney stones in women. Ann Intern Med 1997; 126: 497-504.

8. Bisballe S, ERIKSEn EF, Melsen FmeselKide L, Sorensen OH, Hessov I. Osteopenia and osteomalacia after gastrectomy: Interrelations between biochemical markers of bone remodeling, vitamin D metabolites, and bone histomorphometry. Gut 1991; 32: 13031307.

9. Tsai KS, Wahner HW, OfFord KP, Melton LJ, KUMAR R, RIGGS BL. Effect of aging on vitamine D stores and bone density in women. Calcif Tissue Int 1987; 40: 241-243.

10. MacLaughuin J, Holick MF. Aging decresases the capacity of human skin to produce vitamin D3. J Clin Invest 1985; 76: 1536-1538.

11. Dawson-Hughes B, Harris SS, Krall EA, Dallal GE, Falconer G, GREen CL. Rates of bone loss in postmenopausal women randomly assigned to one of two dosages of vitamin D. Am J Clin Nutr 1995; 61: 1140-1145.

12. Gregg EW, Cauley JA, Seely DG, Ensrud Ke, BAUER DC. Physical activity and osteoporotic fracture risk in older women. Ann Int Med 1998; 129: 81-88.

13. HopPer JL, SEEMAN E. The bone density of female twins discordant for tobacco use. N Engl J Med 1994; 330: 387-391.

14. Byrjalsen I, HaArbo J, Christiansen C. Role of cigarette smoking on the postmenopauseal endometrium during sequential estrogen and progestogen therapy. Obstet Gynecol 1993; 81: 1016-1021.

15. Liberman UA, Weiss SR, BRoll J, MinNe HW, QuAN H, Bell NH et al. Effect of oral alendronate on bone mineral density and the incidence of fractures inn postmenopausal osteoporosis. N Eng J Med 1995; 333: 14371444.

16. Harris ST, Gertz BJ, Genant HK, Eyre DR, SURUILL TT, VENTURA JN et al. The effect of short term treatment withn alendronate on vertebral density and biochemical markers of bone remodeling in early postmenopausal women. J Clin Endocrinol Metab 1993; 76 : 1399-1406.

17. Chesnut CH, McClung MR, EnsRud Ke, Bell NH, GENANT HK, HARRIS ST et al. Alendronate treatment of the postmenopausal osteoporotic woman: efffect of multiple dosages on bone mass and bone remodeling. Am J Med 195; 9: 144-152.
18. Tucci JR, Tonino RP, Emkey RD, Peverly CA, KHER U, SANTORA AC. Effects of three years of oral alendronate treatment in postmenopausal women with osteoporosis. Am J Med 1996; 101: 488-501.

19. Balck DM, Cummings SR, Karpf DB, Cauley JA, Thompson DE, NevitT MC et al. Randomised trial of effect of alendronate on risk of fracture in women with existing vertebral fractures. Lancet 1996; 348: 1535-1542.

20. Hosking D, Chilvers CE, Christiansen C, Raun P, Wasaich R, Ross P et al. Prevention of bone loss with alendronate in postmenopausal women under 60 years of age. N Eng J Med 1998; 338: 485-492.

21. Cummings SR, Black DM, Thompsom DE APPLEGATE WB, BARRET-CONNOR E, MuSLINER TA et al. Effect of alendronate on risk of fracture in women with low bone density but without of vertebral frectures. Results from the Fracture Intervention Trial. JAMA 1998; 280: 2077-2082.

22. TONINo RP, MEUNier PJ, EMKEy R, RodRIGUEZPortales JA, MEnKes CJ, Wasnich RD et al. Skeletal benefits of alendronate: 7-year treatment of postmenopausal osteoporotic women. Phase III Osteoporosis Treatment Study Group. J Clin Endocrinol Metab 2000; 85: 3109-3115.

23. GREenspan SL, Bone G, SChNitZer TJ, Watts NB, ADAMI S, FolDEs AJ et al. Two-year results of once-weekly administration of alendronate $70 \mathrm{mg}$ for the treatment of postmenopausal osteoporosis. J Bone Miner Res 2002; 17: 1988-1996.

24. Greenspan S, Field-Munves E, Tonino R, Smith M, PetruschKe R, WANGL L et al. Tolerability of once-weekly alendronate in patients with osteoporosis: a randomized, double-blind, placebo-controlled study. Mayo Clin Proc 2002; 77: 1044-1052.

25. DE Groen PC, Lubbge DF, HiRsch LJ, Daifotis A, STEPHENSON W, FREEDHOLAN D et al. Esophagitis associated with the use of alendronate. N Eng J Med 1996; 335: 1016-1021.

26. LevinE J, NELSON D. Esophageal structure associated with alendronate therapy. Am J Med 1997; 102: 489-491.

27. HaRris ST, Watts NB, Genant HK, McKeever CD, HANGARTNER T, KELLER $M$ et al. Effects of risedronate treatment on vertebral and nonvertebral fractures in women with postmenopausal osteoporosis: randomized controlled trial Vertebral Efficacy with Risedronate Therapy (VERT) Study Group. JAMA 1999; 282: 1344-1352. 
28. Reginster J, Minne HW, Sorensesn OH, Hooper M, Roux C, BRANDI ML et al. Randomized trial of the effects of risedronate on vertebral in women with established postmenopausal osteoporosis. Vertebral Efficacy with Risedronate Therapy (VERT) Study Group. Osteoportosis Int 2000; 11: 83-91.

29. McClung MR, Geusens P, Miller PD, Zippel H, BENSEN WG, Roux C et al. Effect of risedronate on the risk of hip fracture in elderly women. Hip Intervention Program Study Group. N Eng J Med 2001; 344: 333-340.

30. Watts NB, Josse RG, Hamdy RC, Hughes RA, MANHART MD, BARTON I et al. Risedronate prevents new vertebral fractures in postmenopausal women at high risk. J Clin Endocrinol Metab 2003; 88: 542-549.

31. Lindsay R, Silverman SL, CoOper C, Hanley DA, BARTON I, BROY SB et al. Risk of new vertebral fracture in the year following a fracture. JAMA 2001; 285: 320-323.

32. Cohen S, Levy RM, Keller M, Boling E, EmKey RD, GREENWALD $M$ et al. Risedronate therapy prevents corticosteroid-induced bone loss: a twelve-month, multicenter, randomized, double-blind, placebo-controlled, parallelgroup study. Arthritis Rheum 1999; 42: 23092318.

33. TAgGart H, Bolognese MA, LindSAy R, EtTINGER MP, Mulder H, Josse RG et al. Upper gastrointestinal tract safety of risedronate: a pooled analysis of 9 clinical trials. Mayo Clin Proc 2002; 77: 262-270.

34. Brown JP, KendLER MR, McClung RD, EMKEY JD, ADACHI MA, Bolognese $Z$ et al. The efficacy and tolerability of risedronate once a week for the treatment of postmenopausal osteoporosis. Calcif Tissue Int 2002; 71: 103111.

35. Delmas PD, Buamason NH, Mitiak BH, Rauoux AC, SHAH AS, HuSTER WJ et al. Effects of raloxifene on bone mineral density, serum cholesterol concentrations, and uterine endometrium in postmenopausal women. N Engl J Med 1997; 337: 1641-1647.

36. EtTinger B, Black DM, MitlaK BH, Knickerbocker RK, NiCKelsen T, GenANT HK et al. Reduction of vertebral fracture risk in postmenopausal women with osteoporosis treated with raloxifene : results from a 3-year randomized clinical trial. Multiple Outcomes of Raloxifene Evaluation (MORE). JAMA 1999; 282: 637-645.

37. Delmas PD, Ensrud KE, Adachi JD, Haroer KD, SARKAR S, GENNARI C et al. Efficacy of raloxifene on vertebral fracture risk reduction in postmenopausal women with osteoporosis: four-year results from a randomized clin trial. J Clin Endocrinol Metab 2002; 87: 3609-3617.

38. Chesnut CH, Silverman S, Adriano K, Genant H GiOMONA A, HARRIS S et al. A randomized trial of nasal spray salmon calcitonin in postmenopausal women with established osteoporosis: The prevent recurrence of osteoporotic fractures study. Am J Med 2000; 109: 267-276.

39. Overgatrd K, Agnusdei D, Hansen MA, Maioli E, Christiansen C, Gennari C. Dose-response bioactivity and bioavailability of salmon calcitonin in premenopausal women. J Clin Endocrinol Metab 1991; 72: 344-349.

40. NeEr RM, ARnaud CD, Zanchetta JR, Prince R, GAICH GA, REginSTER JY et al. Effect of parathyroid hormone (1-34) on fractures and bone mineral density in postmenopausal women with osteoporosis. N Eng J Med 2001; 344: 1434-1441.

41. CRandall C. Parathyroid hormone for treatment of osteoporosis. Arch Int Med 2002; 162: 2297-2309.

42. Body JJ, Gaich GA, Scheele WH, Kulkami PM, Miller PD, PERETz A et al. A randomized double-blind trial to compare the efficacy of teriparatide (recombinant human parathyroid hormone 1-34) with alendronate in postmenopausal women with osteoporosis. J Clin Endocrinol Metab 2002; 87: 4528-4535.

43. Meunier PJ, Slosman DO, Delmas PD, Sebert JL, Brandi ML, Albanese C et al. Strontium ranelate: dose-dependent effects in established postmenopausal vertebral osteoporosis, a 2-year randomized placebo controlled trial. J Clin Endocrinol Metab 2002; 87: 2060-2066.

44. Ravn P, Hosking D, Thompson D, Cizza G, WASNICH RD, McCLUNG M et al. Monitoring of alendronate treatment and prediction of effect on bone mass by biochemical markers in the early postmenopausal intervention cohort study. J Clin Endocrinol Metab 1999; 84: 2363-2368.

45. Pharmacological interventions for postmenopausal osteoporosis: an evidencebased approach. Rheumatology 2000; 39: 1309-1315.

46. HäUSELMANN HJ, RizzOLI R. A comprehensive review of treatments for postmenopausal osteoporosis. Osteoporosis Int 2003; 14: 212. 